\title{
ВАЖНОСТЬ ПСИХОЛОГИЧЕСКОГО И ДИЕТОЛОГИЧЕСКОГО НАБЛЮДЕНИЯ ПОСЛЕ БАРИАТРИЧЕСКОЙ ХИРУРГИИ: ОБЗОР ЛИТЕРАТУРЫ
}

\author{
ОРИГИНАЛЬНАЯ СТАТЬЯ
}

DENDASCK, Carla Viana1, SANTOS, Rogério Bongestab dos², SANTOS, Vitor Maia $^{3}$, ANDRADE, Tadeu Uggere de ${ }^{4}$, PFLUG, Adriano Ribeiro Meyer ${ }^{5}$

DENDASCK, Carla Viana. et al. Важность психологического и диетологического наблюдения после бариатрической хирургии: Обзор литературы. Revista Científica Multidisciplinar Núcleo do Conhecimento. Год. 06, Изготовить. 10, Вол. 01, с. 20-44. Октябрь 2021 года. ISSN: 2448-0959, Ссылка

\footnotetext{
${ }^{1}$ Богослов, кандидат клинических психоанализов. В течение 15 лет он работал с научной методологией (методом исследования) в научно-производственной ориентации магистрантов и докторантов. Специалист в области маркетинговых исследований и медицинских исследований, докторант в области коммуникации и семиотики (PUC SP).

${ }^{2}$ Окончила медицинскую школу Санта-Каса-де-Мизерикордия в Витории - EMESCAM. Медицинская ординатура по общей хирургии в больнице Санта-Каса-де-Мизерикордия-де-Витория (HSCMV). Аспирант по диетотерапии в EMESCAM. Аспирант по нутрологии факультета медицинских наук Санта-Каса-деМизерикордиа в Сан-Паулу (FCMSCM-SP / ABRAN). Специалист по нутрологии Бразильской ассоциации нутрологов (ABRAN). Специалист по диетотерапии Бразильского общества парентерального и энтерального питания (SBNPE / BRASPEN).

${ }^{3}$ Психиатр. Специалист по психиатрии и судебной психиатрии Бразильской ассоциации психиатрии (АВР). Врач Министерства экономики (МЭ). Квалифицированный магистр фармацевтических наук Университета Вила-Велья (UVV). Заместитель директора-секретаря Психиатрической ассоциации Эспириту-Санто (APES). Секретарь комиссии по трудовой психиатрии АВР. Профессор аспирантуры по психиатрии в университете Вила-Велья (UVV). Аспирантура в области медицины труда и аспирантура в области медицинской экспертизы.

${ }^{4}$ Докторантура физиологических наук - Федеральный университет Эспириту-Санту, UFES. Магистр международного менеджмента - Берлинский университет Штайнбайса, STW-SIBE. Степень магистра физиологических наук - Федеральный университет Эспириту-Санту, UFES. Специализация в области функциональной гастрономии - Методический колледж Сан-Паулу, FAMESP. Диплом в области фармации - Фармацевтический и биохимический факультет Эспириту-Санту, FAFABES.

${ }^{5}$ Врач FMUSP. Окончила медицинский факультет Университета Сан-Паулу (FMUSP). Медицинская ординатура по общей хирургии и продвинутой общей хирургии в больнице Das Clinicas, медицинский факультет Университета Сан-Паулу (HC-FMUSP). Общий хирург Бразильского колледжа хирургов (CBC), хирург пищеварительной системы Бразильского колледжа пищеварительной хирургии (CBCD). Бариатрическая хирургия (SBCBM) Врач-ассистент отделения III хирургической клиники Центрального института больницы das Clínicas при FMUSP.
}

RC: 98484

Доступно в: https://www.nucleodoconhecimento.com.br/психология/послебариатрической 
доступа:

https://www.nucleodoconhecimento.com.br/психология/после-

бариатрической, DOI: 10.32749/nucleodoconhecimento.com.br/ru/98484

\section{PEЗЮME}

Ожирение является многофакторным хроническим заболеванием. У пациента может быть несколько клинических состояний, связанных с ним. Клиническая картина сложная, что может препятствовать применению обычных методов лечения и методов. Бариатрическая хирургия является эфффективным долгосрочным лечением этого состояния. Основываясь на обзоре литературы, статья направлена на обсуждение важности пост-бариатрического психологического и пищевого наблюдения. Фундаментальный вопрос, который лежит в основе этого исследования: как типичные стратегии в послеоперационном периоде могут способствовать тому, чтобы человек, перенесающий операцию, придавал большее значение его диете и психическому здоровью? Наиболее эфрфективной стратегией лечения была бариатрическая хирургия, однако междисциплинарная оценка, которая оценивает биологический, социальный и психологический статус пациента, является фундаментальной, поскольку психопатологические изменения являются общими для людей с ожирением. Хотя бариатрическая хирургия способствует улучшению качества жизни пациентов, необходимо уделять больше внимания послеоперационному лечению. Этим людям следует рекомендовать продолжать диетологическое и психологическое наблюдение в послеоперационном периоде, чтобы можно было предотвратить возможные осложнения в среднесрочной и долгосрочной перспективе.

Ключевые слова: ожирение, психическое здоровье, мониторинг питания, бариатр.

RC: 98484

Доступно в: https://www.nucleodoconhecimento.com.br/психология/послебариатрической 


\section{1. ВВЕДЕНИЕ}

Ожирение является растущей эпидемией и, таким образом, способствует развитию сопутствующих заболеваний, таких как диабет и системная артериальная гипертензия (RODRIGUES et al., 2017). Это достигает глобальных масштабов, около двух миллиардов человек страдают ожирением или избыточным весом. В 2008 году было подсчитано, что около 43\% взрослого населения страдали ожирением, однако это число увеличилось, достигнув 54\% в 2015 году (RODRIGUES et al., 2017). В Бразилии было установлено, что, согласно данным Системы эпиднадзора за рисками и защитными факторами хронических заболеваний путем телефонного обследования, 53\% взрослого населения считались избыточным весом и 18,9\% страдали ожирением (BRASIL, 2017). С увеличением ожирения количество бариатрических операций, следовательно, увеличилось, как указывает Бразильское общество бариатрической и метаболической хирургии (SBCBM, 2017). Бразилия является второй страной в мире по количеству бариатрических операций, выполняемых ежегодно. По сравнению с 2015 годом количество проведенных операций увеличилось на 7,5\% (SBCBM, 2017). Потеря веса после операции отражает значительное улучшение сопутствующих заболевания, таких как диабет, дислипидемия, гипертония и апноэ во сне (BUCHWALD et al., 2004). Однако следует подчеркнуть, что хирургическое лечение является лишь частью, связанной с интегральным лечением ожирения, а диетологическое и психологическое наблюдение после операции имеет принципиальное значение. Поэтому следует инвестировать в стратегии укрепления здоровья и продольной клинической помощи (BRASIL, 2017). Пациент, который стремится выполнить операцию, должен соответствовать некоторым требованиям, независимо от хирургической техники, таким как ИМТ выше 40 кг / м² или ИМТ выше 35 кг / м² при наличии сопутствующих заболеваний, связанных с избыточным весом (SBCBM, 2006). Список сопутствующих заболевания был расширен, и была рассмотрена большая часть населения (BRASIL, 2017).

$\mathrm{RC}: 98484$

Доступно в: https://www.nucleodoconhecimento.com.br/психология/после-

бариатрической 
Каждый пациент, подвергающийся этому типу операции, должен пройти междисциплинарное наблюдение. Целью является оценка, ориентация и рвение к отношениям команда-пациент, так как это способ повышения эфрфективности лечения в послеоперационном периоде (GORDON; KAIO; SALLET, 2011). Диетолог играет жизненно важную роль в предоперационном и послеоперационном периодах. Консультирование по вопросам питания в предоперационном периоде неразрывно связано с увеличением потенциала успеха в послеоперационном периоде (CRUZ, 2004). Исследование, связанное с рецидивом веса, показало, что у 39\% прооперированных пациентов была прибавка в весе (особенно после 12 месяцев операции). К числу фракторов, поднявших эту проблему, можно отнести низкую посещаемость консультаций по вопросам питания в предоперационный период, кульминацией которых стало рецидив веса (BARDAL; CECATTO; MEZZOMO, 2016). Люди, которые проходят через процесс обучения питанию и консультирования, имеют лучшие результаты.

Психологическое и диетологическое консультирование должно проводиться до и после операции. Регургитация, которая обычно поражает пациентов, которые не имеют правильной привычки жевать, является общей для пациентов, которые не проходят какой-либо тип междисциплинарного наблюдения (COOPER et al., 1999). Тем не менее, существует высокий уровень отсева в послеоперационном питании, что может привести к метаболическим осложнениям и рецидиву веса (MENEGOTTO et al., 2013). В силу этих причин корректировка кормления в предоперационном периоде имеет принципиальное значение, поскольку, таким образом, новые пищевые привычки, обусловленные требованиями послеоперационного периода, будут более благоприятными для практического применения (RODRIGUES et al., 2017). Пожизненные корректировки, которые включают пищевое поведение и ффизическую активность, необходимы для успешной потери веса и предотвращения долгосрочных осложнений (COSTA, 2013).

$\mathrm{RC}: 98484$

Доступно в: https://www.nucleodoconhecimento.com.br/психология/послебариатрической 
В предоперационной оценке питания, помимо полного анамнеза питания, проводится прием пищи, лабораторные анализы, диагностика сопутствующих заболевания и весовой анамнез. Он ценится для коррекции часто выявляемых дефицитов питательных веществ. Пациенты с ожирением иногда подвергаются множественным ограничительным диетам и, таким образом, могут рассматриваться как недоедающих (DOLNE; SOUZA; MATTE, 2020). Тем не менее, следует оценить другие вопросы, относящиеся к хирургии, такие как доступность для изменений и оценка общих знаний о питании и самой операции (AILLS et al., 2008). Такая информация позволяет профессионалу определить возможные барьеры, которые могут помешать потере веса или привести к негативным послеоперационным состояниям, таким как злоупотребление алкоголем и другими наркотиками. Кроме того, пациент должен быть готов к первым фазам эволюции послеоперационной диеты (COSTA, 2013). Таким образом, предоперационное диетологическое просвещение должно способствовать улучшению хирургического исхода.

Психологическая поддержка, оказываемая пациенту, который является кандидатом на бариатрическую хирургию (пред- и послеоперационную), имеет определенные цели (ROCHA; COSTA, 2012). Среди них мы выделяем знания о привычках питания, образе жизни, отношениях с едой и эмоциях, связанных с этими взаимодействиями (KORTCHMAR et al., 2018). После этих знаний психолог становится способным узнать и провести линию преемственности от послеоперационного психологического наблюдения, потому что шансы на достижение успеха при хирургическом лечении выше (SILVA, 2015). Зная фракторы, которые могут породить трудности в этом адаптационном процессе, пациенту обеспечивается большая безопасность и повышенное внимание в этих аспектах, что поставило бы его в диапазон риска увеличения веса (FAGUNDES; CAREGNATO; SILVEIRA, 2016).

Таким образом, психологическая поддержка является способом познания фракторов, которые препятствуют эфрфективности пост-бариатрического

$\mathrm{RC}: 98484$

Доступно в: https://www.nucleodoconhecimento.com.br/психология/после-

бариатрической 
лечения (MENSORIO, 2013). Учитывая этот сценарий, клинический опыт показал, что успех хирургического лечения ожирения зависит не только от технического качества хирургической процедуры. Таким образом, это процесс, который зависит от способности пациента адаптироваться к новому рациону питания, новому телу и новому образу жизни (MAGDALENO JR; CHAIM; TURATO, 2009). Трудности в резком изменении качества, а также в количестве того, что поглощается, могут привести к тому, что люди, перенесшие операцию, не потеряют вес или, если не все, похудеют (KORTCHMAR et al., 2018). Этот процесс может породить чувство неудачи и безнадежности, по этой причине необходима психологическая поддержка.

Долгосрочное поддержание веса имеет важное значение (KULICK; HARK; DEEN, 2010). Еще одним моментом, который следует оценить, является предоперационная потеря веса. Нет достаточных доказательств в поддержку этой потери веса до операции, потому что, когда она сделана, это способствует ненужной задержке лечения пациента (KIM et al., 2016). Исследования утверждают, что предоперационная низкокалорийная диета может вызвать определенные ранее существовавшую нехватку питательных веществ, а также потеря мышечной массы может привести пациента к риску недоедания, однако другие исследования понимают, что уменьшение абдоминального и печеночного объема имеет лучшие долгосрочные результаты (EDHOLM et al., 2011; THIBAULT et al., 2016). В связи с настоящей статьей в настоящей статье в качестве фундаментального вопроса возникает вопрос: как стратегии, характерные для послеоперационного периода, способствуют большей осторожности с диетой и психическим здоровьем у пациентов? Чтобы ответить на него, был проведен обзор литературы с целью обсуждения важности постбариатрического психологического и пищевого наблюдения.

$\mathrm{RC}: 98484$

Доступно в: https://www.nucleodoconhecimento.com.br/психология/после- 


\section{2. ОЖИРЕНИЕ И ЕГО ПОСЛЕДСТВИЯ}

Ожирение значительно снижает качество жизни и способствует предрасположенности к другим неинфекционным заболеваниям, особенно тем, которые наносят вред психическому здоровью человека (MARTINS; MIYAZAKI, 2019). По этой причине ожирение следует рассматривать как проблему общественного здравоохранения, учитывая, что повседневная жизнь людей скомпрометирована.

Чтобы сделать контекст этого исследования более разграниченным, последствия бариатрической хирургии, многопрофильную команду, которая является частью этой вселенной, и уход, предоставляемый психологом и диетологом после операции, необходимо понять многофракторность, которая включает ожирение и, следовательно, бариатрического пациента (GORDON; KAIO; SALLET, 2011). Поэтому изначально именно пациент нуждается в постоянном психологическом слежении, так как выполнение данного вида операции требует изменения привычки не только пациента, но и той социальной группы, частью которой он является (KORTCHMAR et al., 2018).

Примечательно, что семейные и межличностные вопросы, соответствующие аспекты психического здоровья, такие как самооценка, афрфективное равновесие и восприятие реальности, и, наконец, наблюдение со стороны многопрофильной команды являются одними из терминов, которые начинают интегрировать жизнь тучного человека, либо до, либо после хирургического процесса (MARTINS; MIYAZAKI, 2019). Таким образом, необходимо понять, в этом сценарии, социальную функцию, которую берут на себя психолог и диетолог, и насколько важно ее выполнение.

Возросшая распространенность избыточного веса и ожирения в мире обеспокоила учреждения общественного здравоохранения, что привело к увеличению государственных расходов. Однако есть правдоподобное

$\mathrm{RC}: 98484$

Доступно в: https://www.nucleodoconhecimento.com.br/психология/послебариатрической 
обоснование: усилия и готовность средств необходимы в многочисленных исследовательских проектах, посвященных теме ожирения, поскольку оно значительно увеличивается с каждым годом (RASOULI et al., 2007). Цифры не типичны для бразильской действительности, поскольку из-за определенных привычек и моделей питания цифры высоки во всем мире.

Такие страны, как Бразилия, Канада и Франция, имеют дело с высоким процентом людей с избыточным весом (около 40\%). Другие страны, такие как США и Германия, классифицируются таким образом более 65\% населения. С учетом этого сценария понимается, что эта социальная реальность отражается в политико-экономическом сценарии, поскольку перегрузка создается в системах здравоохранения более значительным образом, то есть она инвестируется в профрилактическую помощь, медицинские консультации, лекарства, госпитализации, обследования, операции, среди других возможностей (MARTINS; МИЯДЗАКИ, 2019). Этому росту способствуют другие косвенные проблемы, такие как отсутствие работы, потеря свободного времени, заболеваемость и смертность (MELO, 2011). Исследование Кас; Sichieri e Gigante (2007) определил, что лечение ожирения обходится дешевле, чем борьбу с сопутствующими заболеваниями, которые его порождают. Однако при лечении требуются более эффрективные и современные профилактические меры.

Такие превентивные меры, с тем чтобы быть более эффрективными, должны поощрять определенные виды практики. Среди них необходимо развивать диалог между многочисленными взаимодополняющими практиками между собой, особенно теми, которые связаны с совместной работой психолога и диетолога (GORDON; KAIO; SALLET, 2011). Ожирение должно быть включено в динамический контекст, в котором болезнь существует, сохраняется и создает механизмы, которые начинаются с плохого питания, низкого расхода энергии, сидячего образа жизни и высокого потребления калорий (MARTINS; MIYAZAKI, 2019). Это среда, которая также нуждается в рассмотрении, и, таким образом,

$\mathrm{RC}: 98484$

Доступно в: https://www.nucleodoconhecimento.com.br/психология/после-

бариатрической 
население должно интерпретироваться с интегральной точки зрения, учитывая, что они включены в культуру потребления, которая должна быть реорганизована. Поэтому следует стимулировать привычки, обеспечивающие коллективное благополучие. В этом контексте бариатрическая хирургия возникает и выступает в качестве правдоподобной альтернативы.

Однако бариатрическая аби сама по себе не в состоянии решить эту проблему общественного здравоохранения, которая влияет на качество жизни ряда людей не только с локальной точки зрения, но и глобально. Поэтому психологическое и диетическое наблюдение имеет первостепенное значение, и поэтому следует инвестировать в лечение, основанное на междисциплинарном подходе (GONÇALVES; KOHLSDORF; PEREZ-NEBRA, 2020). Тщательный мониторинг медицинских работников из разных областей и самой семьи имеет важное значение для того, чтобы лечение, которому был представлен пациент, было эффрективным в долгосрочной перспективе. Бариатрическая хирургия не ограничивается только потерей веса (FANDIÑO et al., 2004). Он стремится, прежде всего, к улучшению биопсихосоциального состояния человека, являясь проблемой, которая пронизывает качество жизни (COSTA et al., 2009). Учитывая, что успех бариатрической хирургии зависит, помимо многопрофильной команды, от мотивации пациента, что подразумевает изменение моделей жизни и потребления (CAMPOS et al., 2016).

\section{3. ХАРАКТЕРИСТИКА БАРИАТРИЧЕСКОЙ ХИРУРГИИ}

Ожирение достигает высоких пиков в глобальном масштабе, что привлекло внимание властей, а также требует многочисленных усилий со стороны все более многогранных специалистов и команд, участвующих в его лечении, включая врачей, медсестер, психологов, диетологов и других медицинских работников (MARTINS; MIYAZAKI, 2019). Поэтому мы ищем альтернативы, которые делают лечение заболевания более целесообразным.

$\mathrm{RC}: 98484$

Доступно в: https://www.nucleodoconhecimento.com.br/психология/после-

бариатрической 
Бариатрическая хирургия в этом контексте рассматривается многими пациентами как наиболее эффрективный выход из эффрективной борьбы с ожирением. Однако в послеоперационном периоде необходимо поддерживать вес, так как его преимущества включают, помимо корректировки состава тела, значительное снижение сопутствующих сопутствующих сопутствующих заболевания (FLORES, 2014). Значительно снижается депрессивная и тревожная симптомы, а также наблюдается улучшение полового функционирования, повышение уровня активности и интегральное улучшение качества жизни, связанного со здоровьем (FLORES, 2014).

Что касается бразильского контекста, то число операций, связанных с уменьшением веса, постепенно увеличивается, но выразительно, что подразумевает необходимость уделения большего внимания вопросам здравоохранения и социального благополучия. В ущерб критике и спорам, связанным С бариатрической хирургией, пациенту рекомендуется соответствовать определенным конкретным предпосылкам, прежде чем он сможет быть представлен ей (GORDON; KAIO; SALLET, 2011). В зависимости от состояния пациента, он может или не может стать пригодным для процедуры. Одним из параметров, позволяющих принять это решение, является его последующее наблюдение многопрофильной командой, что также подразумевает наличие семьи и собственной силы воли пациента для изменения привычек и поведения (FANDIÑO et al., 2004). Некоторые сопутствующие заболевания выступают в качестве отягчающих факторов для неафорего бариатрического в качестве альтернативы решению проблемы.

Можно привести некоторые примеры, такие как тяжелая пневмопатия, почечная недостаточность, выраженное повреждение миокарда и цирроз печени (FANDIÑO et al., 2004). Тем не менее, бариатрический вопрос, как правило, хорошо принят и рекомендован. Что касается рекомендаций по бариатрическому вмешательству, то выделяются те, которые связаны с человеком и сопутствующими заболеваниями. В начале 1990-х годов

$\mathrm{RC}: 98484$

Доступно в: https://www.nucleodoconhecimento.com.br/психология/послебариатрической 
Национальный институт здравоохранения США начал подчеркивать важность междисциплинарного подхода в предоперационный период, включая, в этом процессе, психологическую оценку перед операцией, являюсь неотъемлемой частью и для принятия решений (FLORES, 2014). Бразильский бариатрический консенсус и Федеральный совет медицины также рекомендовали эту практику, но не без предварительного определения важности ффигуры психолога и/или психиатра в многопрофильной команде (COUTINHO, 1999).

Как повторяет Федеральный совет по медицине, профессионал, отвечающий априори за лечение психического здоровья, понимаемый здесь как психолог и / или психиатр, является частью команды, которая берет на себя основную ответственность. Он направлен на то, чтобы предоставить пациенту лучшее (критическое) представление о состоянии своего здоровья, что непосредственно способствует улучшению лечения адем (ROSA et al., 2020). Во время предоперационной междисциплинарной оценки, в дополнение к предварительной оценке истории личного и семейного здоровья, команда должна быть обеспокоена, среди прочего, практикой / использованием или отсутствием психоактивных веществ, наличием психотических или медицинских состояний, интеллектуальным и когнитивным уровнем, которые позволяют пациенту осознавать риски, успехи и уход, связанные с хирургическим процессом, которому он будет подвергаться, такие как предоперационный уход, а также внимание следует уделять аспектам, которые влияют на качество жизни пациентов и их семей (FLORES, 2014).

Ввиду вышесказанного, необходимо обсудить классификацию бариатрических операций, которые являются: ограничительными или инвалидсорбционными.

Среди наиболее распространенных - рукавная гастрэктомия, также называемая Sleeve, с ограничительной операцией, при которой удаляется большая часть желудка (дно и тело желудка), оставляя остаточную фрорму рукава желудка (прямая трубка) объемом 150 мл. Пациенты, прошедшие эту процедуру, в

$\mathrm{RC}: 98484$

Доступно в: https://www.nucleodoconhecimento.com.br/психология/послебариатрической 
течение первых 2 лет испытывают снижение общего веса в среднем на 30\% (COUTINHO, 1999).

Техника Capella, известная как желудочный by-pass в $y$-Roux, состоит измодальности, которая собирает ограничение на дисабсорбцию. Это гастропластика, связанная с гастроеюнальным шунтом в виде буквы Ү (называется $y$-Roux).Процедура уменьшает размер желудка настолько, что он способен адаптироваться к объему менее 30 мл (MARTINS; МИЯДЗАКИ, 2019). Это также устанавливает связь с более дистальной кишечной петлей (анастомоз), что приводит к обширной дисаборстивной области, поскольку около 3 метров стройных отклоняются от транзита пищи (FANDIÑO et al., 2004).

Техника скопинаро,имеющая более диссорбтивный характер, представляет собой метод, состоящий из применения частичного билиопанкреатическогообходас дистальной гастрэктомией (FANDIÑO et al., 2004). Поскольку он имеет более недоедающий потенциал и более дисабсорбционный, эта техника была менее выполнена, чем техники Sleeve и Capella.

Цель состоит не в том, чтобы указать на лучшую процедуру, а в том, чтобы перечислить существующие возможности и обсудить преимущества и способы, которые могут привести пациента к лучшему качеству жизни, поскольку, среди прочего, основной целью является содействие снижению веса, способствуя, параллельно, улучшению здоровья бариатрических пациентов.

RC: 98484

Доступно в: https://www.nucleodoconhecimento.com.br/психология/после- 


\section{4. ВАЖНОСТЬ ПОСЛЕДУЮЩЕГО НАБЛЮДЕНИЯ ЗА ПИТАНИЕМ И УМСТВЕННЫМ ПИТАНИЕМ}

\section{1 МОНИТОРИНГ ПИТАНИЯ}

Бариатрическая хирургия - это процедура, которая добавляет положительные результаты к лечению ожирения и связанных с ним сопутствующих заболеваниях. Это тема, которая долгое время обсуждалась преимущественно врачами и диетологами, но сегодня является частью междисциплинарного подхода, включая психологию, психиатрию, эндокринологию, анестезиологию, кардиологию, физиотерапию, сестринское дело и фризическое воспитание (FAGUNDES; CAREGNATO; SILVEIRA, 2016). Учитывая, что эти специалисты имеют важное значение в лечении (TRAVADO et al., 2004).

Исследование Silva et al. (2015) стремился определить профиль лиц, которые решили выполнить бариатрическую хирургию в качестве альтернативы лечению ожирения. Был сделан вывод о том, что из 100 опрошенных $49 \%$ заявили, что они выбрали операцию из-за неудачи предыдущих методов лечения; 39\% в ущерб сопутствующим заболеваниям; и, наконец, 12\% выбрали процедуру из-за ее эффективности и легкости в потере лишнего веса (SILVA et al., 2015).

Цели, которые побудили пациентов обратиться за бариатрической хирургией, указывают на то, что 87\% предпочли желать лучшего качества жизни; $82 \%$ для улучшения здоровья; 34\% для эстетических фракторов; и 15\% для социальных факторов (SILVA et al., 2015). Пациенты, которые прошли шесть месяцев предоперационного наблюдения за питанием, имели большее снижение веса, чем те, кто до операции прекратил терять вес или восстановил потерянный вес (KORTCHMAR et al., 2018). Ввиду этого сценария предполагается, что более длительное время наблюдения за питанием пациентов, которые являются кандидатами на бариатрическую хирургию, может понизить их, что может

RC: 98484

Доступно в: https://www.nucleodoconhecimento.com.br/психология/после-

бариатрической 
оправдать результаты, полученные в двух исследованиях. В этом смысле информация, предоставленная специалистом-диетологом, может способствовать новым привычкам в еде, что увеличивает шансы на успех операции (ARAGÃO; ZAMBON, 2021).

Частота, с которой эти пациенты проводят консультации с диетологами, является решающим фрактором успеха операции. Те, кто проходит это наблюдение, имеют большую потерю лишнего веса после операции (BARDAL; CECCATTO; MEZZOMO, 2016). Рецидив веса наблюдался у 39\% пациентов, обратившихся к восстановительной гастропластике, особенно через 12 месяцев после хирургической процедуры. Что касается предоперационной потери веса, то у 74\% пациентов была представлена потеря веса без нутритивного наблюдения, что обосновывает актуальность этого специалиста в послеоперационном периоде (BARDAL; CECCATTO; MEZZOMO, 2016). Снижение веса в предоперационный период полезно, так как оно повышает придерживание пациентом более здоровых привычек, поскольку он будет более обусловлен адекватной диетой после процедуры. Однако, с другой стороны, снижение веса с помощью необоснованных методов, таких как ограничение диеты и низкокалорийная диета без контроля за питанием, являются одними из аспектов, которые могут вызвать ситуации дефицита питательных веществ, распространенные у пациентов с ожирением (RODRIGUES; FARIA, 2020). Это случай витамина D и профриля железа у женщин детородного возраста (RODRIGUES et al., 2017).

Еще одним фрактором, который следует учитывать при оценке, является уменьшение мышечной массы из-за сильного ограничения калорийности. Это может вызвать нарушения функциональной способности, снижение синтеза белка и снижение иммунной компетентности (BONAZZI et al., 2007). Кроме того, предоперационное снижение веса основано на трансоперационной легкости для хирурга, при которой происходит уменьшение внутрибрюшного жира и уменьшение объема печени, которая у большинства пациентов, желающих

$\mathrm{RC}: 98484$

Доступно в: https://www.nucleodoconhecimento.com.br/психология/после-

бариатрической 
хирургического вмешательства, страдает печеночным сататозом. Другие исследования показывают, что снижение веса более чем на 9,5\% более значительно снижает осложнения по сравнению с потерей веса ниже 5\% у пациентов с ИМТ более 45 кг / м². Пациенты с ИМТ > 50 кг/м² могут извлечь наибольшую пользу с точки зрения хирургической техники (THIBAULT et al., 2016).

Целью наблюдения за питанием перед операцией является повышение осведомленности о важности соблюдения здоровых привычек питания, необходимых для поддержания долгосрочной послеоперационной потери веса (GONÇALVES; KOHLSDORF; PEREZ-NEBRA, 2020). Ввиду этого сценария знания о продуктах питания и питании у лиц, представленных и кандидатов на бариатрическую хирургию, вызывают некоторые частые сомнения, такие как возможность рвоты, удовлетворение в первые 48 часов после операции, возможные изменения вкуса и депрессивные состояния, что еще больше усиливает важность работы многопрофильной команды (NISHIYAMA et al., 2007). Исследование выявило выразительное влияние междисциплинарного наблюдения на эфффективность бариатрической хирургии и продемонстрировало, что жизненно важно подготовить человека перед выполнением операции (MASARI et al., 2012).

Пищевое поведение людей, страдающих ожирением, связано с окружающей средой, в которой живет человек. Неадекватные привычки в еде, приобретенные в детстве, подтверждают повышенную адипозность тела (NISHIYAMA et al., 2007). Кроме того, наблюдается ухудшение качества жизни у лиц с избыточным весом, поскольку существует большая вероятность развития сопутствующих заболевания, связанных с ожирением. Таким образом, мероприятия в области питания, связанные с практикой просвещения по вопросам питания, также способствуют повышению качества жизни (BATISTA FILHO; RISSIN, 2003). Исследование, в котором сравнивали пищевое поведение с предпочтениями лиц в пред- и послеоперационном периоде

$\mathrm{RC}: 98484$

Доступно в: https://www.nucleodoconhecimento.com.br/психология/после-

бариатрической 
бариатрической хирургии, показало, что из пациентов, на которых проводилась предоперационная подготовка, 94\% имели «щипает поведение». Из пациентов, перенесющих операцию, 60\% имели такое же поведение (MACHADO et al., 2008).

Среди пациентов, перенесющих эту хирургическую процедуру, 33,3\% приобрели лучшие привычки в еде после операции, а 63\% остались с теми же привычками, которые были выявлены до операции, что еще больше усиливает важность наблюдения за питанием (RODRIGUES et al., 2017). До операции пациенты отдавали предпочтение высококалорийным продуктам, таким как простые углеводы (42\%), сладости (26\%) и закуски (14\%) (MACHADO et al., 2008). Однако после операции исследование показало, что пациенты искали легко потребляемые продукты, такие как сладости (45\%), закуски (22\%), углеводы (20\%) и повышенное предпочтение пива (7\%) (MACHADO et al., 2008). Частота эпизодов переедания у пациентов до операции увеличивалась из-за повышенной нервозности, что было фактором дисбаланса, стимулирующим избыточное потребление пищи, и 24\% сообщили о наличии тревожных и / или расстройств настроения (депрессии).

Тучные компульсивные люди имеют большую предрасположенность стать «щипать» после бариатрической хирургии, поскольку предпочтение потребления сладостей и закусок выше у компульсивных тучных людей, чем у людей с некомульсивным ожирением (COLLES; DIXON; O'BRIEN, 2008). Привычки к «щипать» и принуждение к еде сами по себе остаются или усиливаются после процедуры, что дополнительно демонстрирует важность психологического и / или психиатрического и пищевого наблюдения в пред- и послеоперационном периодах, поскольку неадекватные привычки могут способствовать увеличению веса у этих пациентов. Они могут быть связаны с более высокой распространенностью желудочно-кишечных симптомов, демпинг-синдрома, а также могут нанести серьезный психологический ущерб

$\mathrm{RC}: 98484$

Доступно в: https://www.nucleodoconhecimento.com.br/психология/послебариатрической 
(FLORES, 2014; MACHADO et al., 2008; GREENBERG; SOGG; PERNA, 2009). Акт еды неразрывно связан с внутренними и внешними раздражителями.

Рассматриваются органические, психические и социальные факторы, и, таким образом, считается, что потребление пищи выходит за рамки аспектов питания и, таким образом, примиряет скрытые мотивы, связанные с пережитыми конфликтами и не зависящие от чувства голода (MACHADO et al., 2008; SOUTO; FERRO-BUCHER, 2006). Ограничение, вызванное бариатрической хирургией, может вызвать риски для компульсивных людей, что может препятствовать их адаптации к новому состоянию питания, поскольку желудочная емкость будет ниже, и, таким образом, принесет вред, если человек продолжит неправильно питаться (BOCCHIERI-RICCIARDI et al., 2006). Люди, которые являются кандидатами на бариатрическую хирургию, понимают динамику, связанную с перееданием. Поиск новых привычек - это способ гарантировать, что новые расстройства не будут преобладать после процедуры. Рекомендации по питанию должны быть разъяснены пациенту.

Темы, имеющие чрезвычайную важность, должны быть рассмотрены таким образом, чтобы был замечен послеоперационный успех, такой как переваривание и всасывание питательных веществ до и после процедуры; рекомендации по приготовлению более здоровых блюд; чтение промышленных этикеток пищевых продуктов; покупки на рынке; и, наконец, эти кандидаты должны быть проинструктированы о кормлении в течение первых месяцев после операции (BOCCHIERI-RICCIARDI et al., 2006). Значение диетолога в течение всего наблюдения в пред- и послеоперационном периоде становится резюме. Для достижения положительных результатов. Предоперационная оценка должна строго соблюдаться, основываясь на ранее разработанном протоколе деятельности. Необходимо, чтобы пациент и междисциплинарная команда были вовлечены и осведомлены о важности продвижения более здоровых привычек питания (MASARI et al., 2012).

$\mathrm{RC}: 98484$

Доступно в: https://www.nucleodoconhecimento.com.br/психология/после-

бариатрической 


\section{2 НАБЛЮДЕНИЕ ЗА ПСИХИЧЕСКИМ ЗДОРОВЬЕМ}

Психологические аспекты могут влиять или быть следствием ожирения, а также могут быть ответственны за увеличение веса или трудности в поддержании веса, достигнутого после операции (VACCARO, 2018). Случаи неспособности поддерживать похудение также связаны с ощущением недостаточной подготовки к необходимым изменениям. Избежание неудачи бариатрического лечения зависит от предоперационного исследования по анамнезу пациента с пищей, от роли пищи в его жизни, а также от необходимости исследовать и заботиться о импульсе, который приводит к прожорливости приема пищи (EDLER, 2017). Хирургия способна похудеть по телу, однако некоторыми психологическими проблемами можно пренебречь, и они связаны с контролем диеты, самооценкой и седанием, связанным с эмоциями, что подразумевает создание идентичности с телом, новый вызов, потому что самооценка, после похудения, является отрицательной (FAGUNDES; CAREGNATO; SILVEIRA, 2016).

Эта негативная конструкция связана с образом тела, избытком кожи, восприятием себя в новом теле и адаптацией и восстановлением и самооценкой в постном теле (ALEGRÍA; LARSEN, 2013). Последующее наблюдение и психологическая поддержка до и после операции способствует улучшению результатов пациентов в их задаче похудеть, получить более высокое качество жизни и уменьшить желание есть, являясь этими решающими фракторами, чтобы избежать рецидива ожирения (HOLLYWOOD; OGDEN; PRING, 2012). Также связано снижение чувства голода, малейшее беспокойство по поводу пищи, а также ощущение большего контроля в отношении приема пищи.

Одно исследование показало, что тип преодоления, принятый пациентом после бариатрической операции, определяет или не определяет результат потери веса, и что психологические и фризиологические фракторы объединяются и

$\mathrm{RC}: 98484$

Доступно в: https://www.nucleodoconhecimento.com.br/психология/после-

бариатрической 
взаимодействуют значительно, тем самым влияя на результаты (например, построение более позитивного образа на теле) (FIGURA et al., 2015).

Прооперированные люди могут пройти через период эмоциональной перестройки, фразу, в которой важно поддерживать психологическое наблюдение с помощью терапии, чтобы избежать когнитивных искажений и восприятия деформированной реальности. (MAGDALENO JR.; CHAIM; TURATO, 2008). С учетом этого сценария обращается внимание на необходимость индивидуализированного психологического лечения в пред- и послеоперационном периоде. Это связано с выявлением и лечением психической структуры каждого пациента, стремясь, с этой осторожностью, избежать дальнейших осложнений после операции (GORDON; KAIO; SALLET, 2011). Физический, психологический, социальный и клинический опыт пациентов, перенесших бариатрическую хирургию, влияет на процесс лечения. Среди тех, которые больше всего влияют на эти предметы: фризические изменения и последующие проблемы, психологический опыт, социальная поддержка и клинический опыт (LIU; IRWIN, 2017).

Наблюдается улучшение психологических, физических и социальных условий, однако существует постоянная озабоченность по поводу возможных непредвиденных событий, характерных для результатов операции, и важность поддержки медицинских услуг, рекомендованных на послеоперационный период, включая, в этом процессе, психологическую поддержку, поскольку пациентам необходимо научиться справляться с изменениями в организме; общественные отношения и конструение новой идентичности (LIU; IRWIN, 2017). Родственники или опекуны бариатрического пациента также нуждаются в психологической поддержке (SPRENGEL, 2015), поскольку социальная поддержка всех участников подразумевает лучшее соблюдение более здорового поведения в отношении здоровья, такого как фризическая активность, более здоровое питание, снижение стрессовых ситуаций (MARTINS; MIYAZAKI,

$\mathrm{RC}: 98484$

Доступно в: https://www.nucleodoconhecimento.com.br/психология/послебариатрической 
2019). Таким образом, поощряется улучшение здоровья, включая психическое здоровье и качество жизни (ABREU RODRIGUES; SEIDL, 2015).

Тем не менее, эфффективность лечения требует некоторой осторожности, такой как время психологической подготовки до и после операции, как для пациента, так и для супругов и членов семьи, поскольку именно они будут сопровождать их на протяжении всего пред- и пост-бариатрического лечения (DELAPRIA, 2019). Мотивация и долгосрочная поддержка пациентов должны стимулироваться в процессе психологического наблюдения (MARCHESINI, 2010). В этом путешествии включение как семьи, так и друзей в предоперационный процесс имеет первостепенное значение, а также обеспечение ясности пациента в отношении выгод и потерь, связанных с процедурой (VACCARO, 2018). Поэтому лечение требует работы многопрофильной команды. При этом следует понимать субъективность каждого случая, предвидя и сопровождая те фракторы, которые прямо или косвенно могут повлиять на успех или неудачу процесса, что подразумевает изменение размера определенных стратегий для достижения успеха (MARCELINO; PATRÍCIO, 2011).

Бариатрическая хирургия, по сути, предлагает пациенту более высокое качество жизни, а также способствует улучшению социализации за счет потери веса, однако следует подчеркнуть, что в этом процессе лечения пациенту все равно придется иметь дело с психическими и пищевыми проблемами, чтобы достичь желаемого качества жизни (DELAPRIA, 2019). Поддержка необходима для того, чтобы можно было сделать возможными изменения в пищевом поведении и образе жизни, а также изменения в теле, социальных сетях и идентичности (VACCARO, 2018). Поддержание и непрерывность веса благодаря хирургическому лечению зависят от действий, осведомленности и приверженности пациента, что может быть трудно и неосуществимо без психологического и пищевого наблюдения.

$\mathrm{RC}: 98484$

Доступно в: https://www.nucleodoconhecimento.com.br/психология/послебариатрической 
Пациент сам должен осознать, что он является трансформировающим агентом. Хирургия худеет, но худое тело зависит от внутренних изменений, требование, которое выходит за рамки желудка. Адаптация к новым измерениям тела и корректировка социальных отношений, а также построение новой идентичности и отключение импульса к еде - это вопросы, которые могут возникнуть после операции, и поэтому требуют большего внимания и заботы (GORDON; KAIO; SALLET, 2011). Психотерапия может способствовать тому, чтобы ум следил за процессом похудения тела.

\section{5. ОКОНЧАТЕЛЬНЫЕ СООБРАЖЕНИЯ}

Это исследование было направлено на демонстрацию важности питания и психического наблюдения до и после бариатрической хирургии. Данные свидетельствуют о том, что роль, которую играет психолог в многопрофильной команде, особенно в послеоперационном периоде бариатрических пациентов, в отношении контроля и / или уменьшения тревожных и депрессивных симптомов и других психопатологических изменений, которые наносят вред психическому здоровью, является существенной, что делает дискуссию о роли психологов и других специалистов, вовлеченных в нее, фундаментальной. Актуальность этих специалистов связана с тем, что мультидисциплинарное действие заставит пациента, представленного в бариатр, привыкнуть к новому уровню жизни (пищевым привычкам, отношениям с пищей и организмом) и в этом процессе психологическое наблюдение в предоперационный и послеоперационный период имеет значение, чтобы избежать таких проблем, как увеличение веса.

Психологическая оценка бариатрических пациентов актуальна, поскольку, таким образом, можно понять этого пациента целостно, и, таким образом, способствует уменьшению психических осложнений от операции, которые могут ухудшить качество жизни пациента. Необходимость углубленной психологической оценки становится суммарной. Пациент должен быть исследован и обучен, чтобы последовательные изменения в хирургии были

$\mathrm{RC}: 98484$

Доступно в: https://www.nucleodoconhecimento.com.br/психология/после-

бариатрической 
эфффективными и визуализированными в долгосрочной перспективе. Показания и противопоказания, связанные с бариатрической хирургией, должны уважать оценку многопрофрильной команды, учитывая, что из-за сборов и давления повседневной жизни проблемы, влияющие на психическое здоровье, такие как тревожные расстройства, такие как тревожные расстройства, среди которых выделяются социальная фобия, генерализованная тревога и паническое расстройство, что касается расстройств настроения, то все большее число людей, страдающих депрессией, вызывает беспокойство.

Такие расстройства стали частыми и преследуют жизнь многих людей. Именно в разгар этого процесса, в некотором роде, турбулентного, обнаруживается пост-бариатрический пациент, которому почти всегда приходится иметь дело с эмоциональной нагрузкой, выходящую за рамки терпимой, что в конечном итоге создает неожиданные расстройства, связанные с тревогой. В этом смысле психолог играет очень важную роль с самого начала хирургического процесса, но его работоспособность не ограничивается им. Поэтому его вклад в послеоперационный период имеет существенное значение.

\section{ссылки}

ABREU-RODRIGUES, M.; SEIDL, E. M. F. Apoio social e reganho de peso póscirurgia bariátrica: estudo de caso sobre intervenção com cuidador. Temas em Psicologia, v. 23, n. 4, p. 1003-1016, 2015.

\section{AILLS, L. et al. ASMBS allied health nutritional guidelines for the surgical} weight loss patient: surgery for obesity and related diseases. Surg Obes Relat Dis., v. 4, n. 5, p. $73-108,2008$.

ALEGRÍA, C. A.; LARSEN, B. "That's who I am: A fat person in a thin body": Weight loss, negative self-evaluation, and mitigating strategies following weight loss surgery.

$\mathrm{RC}: 98484$

Доступно в: https://www.nucleodoconhecimento.com.br/психология/послебариатрической 
Journal of the American Association of Nurse Practitioners, v. 27, n. 3, p. 137144,2013

ARAGÃO, C. N.; ZAMBON, E. A avaliação psicológica para cirurgia bariátrica - uma análise teórica e prática. Rumos da inFormação, v. 2, n. 1, p. 63-76, 2021.

BARDAL, A. G.; CECCATTO, V.; MEZZOMO, T. R. Fatores de risco para recidiva de peso no pós-operatório tardio de cirurgia bariátrica. Scientia médica, v. 26, n. 4, p. ID24224-ID24224, 2016.

BATISTA FILHO, M.; RISSIN, A. A transição nutricional no Brasil: tendências regionais e temporais. Cadernos de saúde pública, v. 19, p. S181-S191, 2003.

BOCCHIERI-RICCIARDI, L. E. et al. Pre-surgery binge eating status: effect on eating behavior and weight outcome after gastric bypass. Obesity surgery, v. 16, n. 9, p. 1198-1204, 2006.

BONAZZI, C. L. et al. A intervençâo nutricional no pré e pós operatório da cirurgia bariátrica. RBONE-Revista Brasileira de Obesidade, Nutrição e Emagrecimento, v. 1, n. 5, p. 59-69, 2007.

BRASIL. Ministério da Saúde. Vigitel 2016: vigilância de fatores de risco e proteção para doenças crônicas por inquérito telefônico. Brasília: Ministério da Saúde, 2017.

BUCHWALD, $\mathrm{H}$. et al. Bariatric surgery: a systematic review and metaanalysis. Jama, v. 292, n. 14, p. 1724-1737, 2004.

CAMPOS, J. et al. O papel da cirurgia metabólica para tratamento de pacientes com obesidade grau ie diabete tipo 2 não controlados clinicamente. ABCD: Arquivos Brasileiros de Cirurgia Digestiva (São Paulo), v. 29, p. 102-106, 2016.

RC: 98484

Доступно в: https://www.nucleodoconhecimento.com.br/психология/после- 
COLLES, S. L.; DIXON, J. B.; O'BRIEN, P. E. Loss of control is central to psychological disturbance associated with binge eating disorder. Obesity, v. 16, n. 3, p. $608-614,2008$.

COOPER, P. L. et al. Nutritional consequences of modified vertical gastroplasty in obese subjects. International journal of obesity, v. 23, n. 4, p. 382-388, 1999.

COSTA, A. C. C. et al. Obesidade em pacientes candidatos a cirurgia bariátrica. Acta Paulista de enfermagem, v. 22, p. 55-59, 2009.

COSTA, D. Eficiência do acompanhamento nutricional no pré e pós-operatório da cirurgia bariátrica. RBONE-Revista Brasileira de Obesidade, Nutrição e Emagrecimento, v. 7, n. 39, 2013.

COUTINHO, W. Consenso latino-americano de obesidade. Arquivos Brasileiros de Endocrinologia \& Metabologia, v. 43, n. 1, p. 21-67, 1999.

DELAPRIA, A. M. T. A importância do acompanhamento psicológico no pré e pósoperatório da cirurgia bariátrica. Revista UNINGÁ, v. 56, n. S1, p. 78-88, 2019.

DOLNE, F.; SOUZA, J. S. M. de.; MATTE, J. Atuação da enfermagem na assistência prestada ao paciente obeso mórbido no pós-operatório de cirurgia bariátrica. RBONE-Revista Brasileira De Obesidade, Nutrição E Emagrecimento, v. 14, n. 87, p. 550-559, 2020.

EDHOLM, D. et al. Preoperative 4-week low-calorie diet reduces liver volume and intrahepatic fat and facilitates laparoscopic gastric bypass in morbidly obese. Obesity surgery, v. 21, n. 3, p. 345-350, 2011.

EDLER, S. Tempos Compulsivos: a busca desenfreada pelo prazer. Rio de Janeiro: Casa da Palavra, 2017. 176 pgns.

$\mathrm{RC}: 98484$

Доступно в: https://www.nucleodoconhecimento.com.br/психология/после-

бариатрической 
FAGUNDES, M. A. B. G.; CAREGNATO, R. C. A.; SILVEIRA, L. M. de. O. B. Variáveis psicológicas associadas à cirurgia bariátrica. Aletheia, v. 49, n. 2, p. 47$54,2016$.

FANDIÑO, J. et al. Cirurgia bariátrica: aspectos clínico-cirúrgicos e psiquiátricos. Revista de psiquiatria do Rio Grande do Sul, v. 26, n. 1, p. 47-51, 2004.

FIGURA, A. et al. Determinants of weight loss following laparoscopic sleeve gastrectomy: the role of psychological burden, coping style, and motivation to undergo surgery. Journal of obesity, v. 2015, p. 1-10, 2015.

FLORES, C. A. Avaliação psicológica para cirurgia bariátrica: práticas atuais. ABCD: Arquivos Brasileiros de Cirurgia Digestiva (São Paulo), v. 27, p. 59-62, 2014.

GONÇALVES, S. J. B. G.; KOHLSDORF, M.; PEREZ-NEBRA, A. R. Adesão ao pósoperatório em cirurgia bariátrica: análise sistemática da literatura brasileira. Psicologia Argumento, v. 38, n. 102, p. 626-646, 2020.

GORDON, P. C.; KAIO, G. H.; SALLET, P. C. Aspectos do acompanhamento psiquiátrico de pacientes obesos sob tratamento bariátrico: revisão. Archives of Clinical Psychiatry (São Paulo), v. 38, p. 148-154, 2011.

GREENBERG, I.; SOGG, S.; PERNA, F. M. Behavioral and psychological care in weight loss surgery: best practice update. Obesity, v. 17, n. 5, p. 880-884, 2009.

HOLLYWOOD, A.; OGDEN, J.; PRING, C. The impact of a bariatric rehabilitation service on weight loss and psychological adjustment-study protocol. BMC Public Health, v. 12, n. 1, p. 1-5, 2012.

KAC, G.; SICHIERI, R.; GIGANTE, D. P. Epidemiologia nutricional. São Paulo: Editora Fiocruz, 2007. 580 pgns.

$\mathrm{RC}: 98484$

Доступно в: https://www.nucleodoconhecimento.com.br/психология/после- 
$\mathrm{KIM}$, J. et al. ASMBS updated position statement on insurance mandated preoperative weight loss requirements. Surgery for Obesity and Related Diseases, v. 12 , n. 5, p. 955-959, 2016.

KORTCHMAR, E. et al. Reganho de peso após a cirurgia bariátrica: um enfoque da fenomenologia social. Acta Paulista de Enfermagem, v. 31, n. 4, p. 417-422, 2018.

KULICK, D.; HARK, L.; DEEN, D. The bariatric surgery patient: a growing role for registered dietitians. Journal of the American Dietetic Association, v. 110, n. 4, p. 593-599, 2010.

LIU, R. H.; IRWIN, J. D. Understanding the post-surgical bariatric experiences of patients two or more years after surgery. Quality of Life Research, v. 26, n. 11, p. 3157-3168, 2017.

MACHADO, C. E. et al. Compulsão alimentar antes e após a cirurgia bariátrica. ABCD: Arquivos Brasileiros de Cirurgia Digestiva (São Paulo), v. 21, p. 185-191, 2008.

MAGDALENO JR, R.; CHAIM, E. A.; TURATO, E. R. Características psicológicas de pacientes submetidos à cirurgia bariátrica. Revista de Psiquiatria do Rio Grande do Sul, v. 31, p. 73-78, 2009.

MAGDALENO JR, R.; CHAIM, E. A.; TURATO, E. R. Psychological characteristics of patients submitted to bariatric surgery. Revista de Psiquiatria do Rio Grande do Sul, v. 31, p. 73-78, 2009.

MARCELINO, L. F.; PATRICIO, Z. M. A complexidade da obesidade e o processo de viver após a cirurgia bariátrica: uma questão de saúde coletiva. Revista Ciência e Saúde Coletiva, v. 16, n. 12, p. 4767-4776, 2011.

$\mathrm{RC}: 98484$

Доступно в: https://www.nucleodoconhecimento.com.br/психология/после-

бариатрической 
MARCHESINI, S. D. Late psychological follow-up on patients submitted to bariatric surgery. ABCD: Arquivos Brasileiros de Cirurgia Digestiva, v. 23, n. 2, p. 108$113,2010$.

MARTINS, C. E. P.; MIYAZAKI, E. T. A presença do psicólogo na equipe multidisciplinar e a ansiedade pós-cirurgia bariátrica. Revista Campo do Saber, v. 5, n. 2, p. 77-90, 2019.

MASARI, S. P. et al. Preparação multidisciplinar pré-cirúrgia bariátrica na visão do cliente. RBONE-Revista Brasileira de Obesidade, Nutrição e Emagrecimento, v. 6, n. 32, p. 1-13, 2012.

MELO, M. E. Os Números da Obesidade no Brasil: VIGITEL 2009 e POF 20082009. 2011. Disponível

em: https://www.saudedireta.com.br/docsupload/1340368729Obesidade\%20no\%20Brasil \%20VIGITEL\%202009\%20POF2008_09\%20\%20II.pdf. Acesso em: 02 ago. 2021.

MENEGOTTO, A. L. S. et al. Avaliação da frequência em consultas nutricionais dos pacientes após cirurgia bariátrica. ABCD: Arquivos Brasileiros de Cirurgia Digestiva (São Paulo), v. 26, p. 117-119, 2013.

MENSORIO, M. S. Análise de estratégias de enfrentamento, ansiedade e hábitos em pacientes elegíveis à cirurgia bariátrica, com e sem acompanhamento psicológico. 2013. 159f. Dissertação (Mestrado em Processos de Desenvolvimento Humano e Saúde) - Universidade de Brasília, Brasília, DF, 2013.

NISHIYAMA, M. F. et al. Avaliação do nível de conhecimento e aderência da conduta nutricional em pacientes submetidos e candidatos à cirurgia bariátrica. Arquivos de Ciências da Saúde da UNIPAR, v. 11, n. 2, p. 89-98, 2007.

RC: 98484

Доступно в: https://www.nucleodoconhecimento.com.br/психология/после-

бариатрической 
RASOULI, N. et al. Ectopic fat accumulation and metabolic syndrome. Diabetes, Obesity and Metabolism, v. 9, n. 1, p. 1-10, 2007.

ROCHA, C.; COSTA, E. Aspectos psicológicos na obesidade mórbida: Avaliação dos níveis de ansiedade, depressão e do auto-conceito em obesos que vão ser submetidos à cirurgia bariátrica. Analise psicológica, v. 30, n. 4, p. 451-466, 2012.

RODRIGUES, G. et al. Acompanhamento nutricional no pré-operatório de cirurgia bariátrica: tempo de seguimento versus redução de peso. Revista PsicoFAE: Pluralidades em Saúde Mental, v. 6, n. 2, p. 97-112, 2017.

RODRIGUES, J. O.; FARIA, H. M. C. Os aspectos psicossociais da cirurgia bariátrica: do pré ao pós-operatório. Cadernos de Psicologia, v. 2, n. 4, p. 551-569, 2020.

ROSA, T. M. F. et al. Atendimento nutricional em grupo de pacientes candidatos a cirurgia bariátrica: repercussões no consumo alimentar. RBONE-Revista Brasileira De Obesidade, Nutrição E Emagrecimento, v. 14, n. 87, p. 690-698, 2020.

SILVA, M. A da. Emagrecimento induzido por cirurgia: ação do psicólogo após a cirurgia bariátrica. Bol. Psicol., v. 65, n. 143, p. 243-246, 2015.

SILVA, P. T. da. et al. Perfil de pacientes que buscam a cirurgia bariátrica. ABCD: Arquivos Brasileiros de Cirurgia Digestiva (São Paulo), v. 28, p. 270-273, 2015.

SOCIEDADE BRASILEIRA DE CIRURGIA BARIÁTRICA E METABÓLICA (SBCBM). 2017. Disponível em: http://www.sbcbm.org.br/. Acesso em: 02 ago. 2021.

SOUTO, S.; FERRO-BUCHER, J. S. N. Práticas indiscriminadas de dietas de emagrecimento e o desenvolvimento de transtornos alimentares. Revista de Nutrição, v. 19, p. 693-704, 2006.

RC: 98484

Доступно в: https://www.nucleodoconhecimento.com.br/психология/после-

бариатрической 
SPRENGEL, A. L. Cirurgia Bariátrica: Manual de instruções para pacientes e familiares. São Paulo: M. Books do Brasil, 2015. 96 pgns.

THIBAULT, R. et al. Twelve key nutritional issues in bariatric surgery. Clinical nutrition, v. 35, n. 1, p. 12-17, 2016.

TRAVADO, L. et al. Abordagem psicológica da obesidade mórbida: Caracterização e apresentação do protocolo de avaliação psicológica. Análise psicológica, v. 22, n. 3, p. 533-550, 2004.

VACCARO, R. V. Acompanhamento psicológico e manutenção da perda de peso após a cirurgia bariátrica do tipo Bypass. 2018. 81f. Dissertação (Mestrado em Psicologia Clínica) - Pontifícia Universidade Católica de São Paulo, São Paulo, SP, 2018.

Опубликовано: октябрь 2021 г.

Утверждено: октябрь 2021 г.

RC: 98484

Доступно в: https://www.nucleodoconhecimento.com.br/психология/после- 